Journal of Mathematics and Informatics

Vol. 19, 2020, 23-35

ISSN: 2349-0632 (P), 2349-0640 (online)

Published 21 July 2020

www.researchmathsci.org

DOI: http://dx.doi.org/10.22457/jmi.v19a04174

Journal of

Mathematics and

Informatics

\title{
A Review of the Mathematical Models for Brucellosis Infectiology and Control Strategies
}

\author{
Nkuba Nyerere ${ }^{1,2^{*}}$, Livingstone S. Luboobi ${ }^{1,3}$, Saul C. Mpeshe ${ }^{4}$ and \\ Gabriel M. Shirima ${ }^{5}$
}

${ }^{1}$ Department of Applied Mathematics and Computational Sciences, Nelson Mandela African Institution of Science and Technology, P.O. Box 447, Arusha, Tanzania. e-mail: nyereren@nm-aist.ac.tz

${ }^{2}$ Department of Mathematics, Informatics and Computational Sciences, Sokoine University of Agriculture, P.O.Box 3038, Morogoro. e-mail: emmankuba@ sua.ac.tz

${ }^{3}$ Institute of Mathematical Sciences, Strathmore University, PO Box 59857-00200, Nairobi, Kenya. e-mail: 1luboobi@strathmore.edu

${ }^{4}$ Department of Mathematics, University of Iringa, P.O.Box 200, Iringa, Tanzania. e-mail: mpesaul@yahoo.com

${ }^{5}$ Department of Global Health and Bio-Medical Sciences, Nelson Mandela African Institution of Science and Technology, P.O. Box 447, Arusha, Tanzania. e-mail: gabriel.shirima@nm-aist.ac.tz

"Corresponding author

Received 2 June 2020; accepted 3 July 2020

Abstract. Brucellosis is a zoonotic bacterial infection that can be acquired by humans from infected animals' meat, urine, body fluids, aborted materials, unpasteurized milk, and milk products or contaminated environment. Mathematical models for infectious diseases have been used as important tools in providing useful information regarding the transmission and effectiveness of the available control strategies. In this paper, a review of the available compartmental mathematical models for Brucellosis was done. The main purpose was to assess their structure, populations involved, the available control strategies and suitability in predicting the disease incidence and prevalence in different settings. Diversities have been observed in the reviewed mathematical models; some models incorporated seasonal variations in a single animal population, some ignored the contributions of the contaminated environment while others considered the cattle or sheep population only. Most of the models reviewed have not considered the contribution of wild animals in the dynamics of Brucellosis. Some models do not match the real situation in most affected areas like sub-Saharan African region and Asian countries where wild animals, cattle and small ruminants share grazing areas and water points. Thus, to avoid unreliable results, this review reveals the need to affirm and incorporate wild animals, livestock, humans and seasonal weather parameters in the spread of Brucellosis and in planning for its controls. 
Nkuba Nyerere, Livingstone S. Luboobi, Saul C. Mpeshe, and Gabriel M. Shirima

Keywords: Brucellosis, infectiology, control strategies, mathematical models, compartmental models

\section{AMS Mathematics Subject Classification (2010): 03H10}

\section{Introduction}

Brucellosis is a disease caused by a number of species of bacteria collectively called Brucella. It is one of the most widespread human and livestock diseases acquired from infected animals or their products [1]. The disease is one of the highest priority animal diseases in different regions of the world. Brucellosis infects many species including livestock, wildlife, and humans. Most human infections are acquired through direct contact with infectious livestock or via indirect transmission by ingestion of unpasturized milk and milk products or through engaging in occupational activities. The disease has a wide range of impacts that include losses due to abortion, culling of infected animals, lost milk production, animal replacement costs, veterinary fees, and human illness- causing reduced work capacity.

The history of Brucellosis goes beyond the 1887 isolation and identification of Brucella melitensis and extends back to people's initial contact with animals [1, 2, 3]. The disease has been known by various names including Malta fever, Crimean fever, Bang's disease, Rock fever, Maltese fever, Gibraltar fever, Undulant fever, Mediterranean fever, or Gastric remittent [4,5]. It remains the most common and continually-reemerging zoonosis worldwide since 1884, and it is accountable for more than 500,000 new human cases annually [6]. Additionally, low infectious doses (10-100 bacterial cells), rapid transmission through different pathways, persistence in the environment, and treatment difficulties with antibiotics make Brucella a possible bioterrorism agent [5].

Control and eradication programmes based on various strategies have been successful in eliminating Brucellosis in several countries [7]. Strategies based on prevention of the spread between animals, monitoring of Brucellosis-free herds and zones, elimination of infected animals by test and slaughter, strict control of the movement of infected and suspected animals, mass immunization to reduce infection rate and supporting specific education and training programmes have all received attention in various countries $[8,9]$. In addition, human treatment is given to cure or reduce symptoms duration, avert relapse, and avoid complications such as encephalitis, spondylitis, arthritis, endocarditis, sacroilitis, epididymoorchitis, and abortion [10]. However, the control or eradication of Brucellosis is highly dependent on national strategies, priorities, and policies.

The current review evaluates different Brucellosis models based on structure, various control interventions, and their effectiveness

\section{Methodology}

A literature search from Medline, Science Direct, OVID, PubMed, Research4Life, and Web of Science databases was conducted and analysis of the articles relevant to the study and written in English was done. The search was restricted to articles published from 2009 to 2019. To minimize the chance of missing important studies, manual search and sorting of the references of all articles found in our search including any potentially eligible studies that were found using Google Scholar were performed. The search keywords or MeSH were defined as: ("Brucellosis" OR "Brucella melitensis" OR 
A Review of the Mathematical Models for Brucellosis Infectiology and Control Strategies

"Brucella abortus" OR "human brucellosis") AND ("mathematical model" OR "modeling" OR "mathematical approach" OR "deterministic model" OR "compartmental model"). Brucellosis studies which were conducted using compartmental models, available in full text, most recent in a series of articles with the same first authors, similar modeling structure and content, and those used to evaluate the potential impact of Brucellosis transmission control strategies on a population-based level were eligible for inclusion in the review. The initial number of returned articles from the searches was 125 . Abstract of articles with compartmental models were retained for a detailed review and 20 were included in the list of references.

\section{Mathematical models}

A mathematical model is an illustration of a system using mathematical language and concepts; it is an essential tool for evaluating the spread and control of communicable diseases. Mathematical models are used to investigate and predict how communicable diseases advance, demonstrate the possible outcomes of an epidemic and advise public health interventions. Such models use some basic assumptions and mathematics to identify the parameters for various infectious diseases and gauge the effects of different interventions such as mass vaccination and education campaign programmes. They can also assist in decisions involving intervention(s) to be implemented and to what extent. Modeling of infectious diseases is a tool that has been used to study the spread of diseases, predicting outbreaks and appraising the control strategies of an epidemic.

In 1766, a trained physician Daniel Bernoulli performed the earliest mathematical modeling study on the spread of diseases. He developed a mathematical model to study smallpox mortalities in England and used the model to show that universal inoculation against the disease would increase life expectancy at birth by 3 years and 2 months [11]. In 1772, Lambert and Laplace improved Bernoulli's work by expanding the model to include age-dependent factors. Nevertheless, this research focus was not systematically developed until 1911 when a benchmark study by Ross established the modern mathematical epidemiology [12]. The law of mass action was then applied by William Hamer and Ronald Ross to explain the epidemic behaviour of measles and malaria respectively. Kermack-McKendrick and the Reed-Frost epidemic models of 1927 and 1928 respectively both described the association between immune, infected, and susceptible individuals in a population. The models successfully predicted the behaviour of outbreaks similar to recorded observations of many epidemics [13].

More importantly, the work by Ross, Kermack, and McKendrick addressed the use of mass-action incidence in disease transmission, signifying that the probability for a susceptible individual to be infected corresponds to the number of its interactions with infectious ones. Furthermore, their work establishes the compartmental deterministic epidemic modeling and predicted the critical fraction of susceptible individuals that must be exceeded in the population for an epidemic to occur depending on the transmission potential of an infection. Forty years after the reports by Ross, MacDonald extended Ross's model to clarify the malaria transmission process and proposed methods for eliminating the disease on an operational level. The significant contribution of MacDonald and Ross to the field through the use of computers made mathematical models for the dynamics and control of mosquito-transmitted pathogens to be branded as 
Nkuba Nyerere, Livingstone S. Luboobi, Saul C. Mpeshe, and Gabriel M. Shirima

Ross-MacDonald models [14]. Generally, there have been several different model compartment structures depending on the type of disease. Some possible classes of compartmental models are: susceptible-infectious (SI), susceptible-infectious-susceptible (SIS), susceptible-exposed-infectious (SEI), susceptible-exposed-infectious-susceptible (SEIS), susceptible-infectious-recovered (SIR), susceptible-infectious-recoveredsusceptible (SIRS), susceptible-exposed-infectious-recovered (SEIR) and susceptibleexposed-infectious-recovered-susceptible (SEIRS) [15].

\section{Brucellosis mathematical models}

Amaku et al. [16] formulated a mathematical model to simulate Brucellosis dynamics in herds of female cattle and analyzed the effects of vaccination strategies. The model was based on statistics from some states of Brazil where surveys on serology were carried out and detected a prevalence rate higher than $2 \%$. The findings showed that in areas with low immunization coverage (approximately 30\%), the time required to lower the prevalence rate to $2 \%$, would be approximately twice the time observed for higher coverage (approximately 90\%). The study further predicted that, if the model parameters remain unchanged, it will take a decade to reduce the Brucellosis prevalence to 1 or $2 \%$ which is the adequate phase for the disease eradication. The intensification of female bovine vaccination efforts to achieve high vaccination coverage was recommended in this study.

Ainseba et al. [17] formulated and analyzed a susceptible-infected mathematical model for Brucellosis in the ovine population. In this model, both direct and indirect modes of disease transmission were considered. Susceptible individuals could directly contract Brucella from infected individuals through contact or indirectly due to the presence of virulent organisms in the environment. The net reproduction number was computed and used to analyze the global asymptotic behaviour of the model. Numerical simulations were performed to investigate the effect of a slaughtering policy. Findings from the study suggested that, in order to better understand the dynamics of the disease in ovine or any other animal population, incorporation of the age of infection and/or the chronological age is needed. However, the model formulated was too elementary to generalize the complex dynamics of the disease that involves wild animals, livestock, human and the environment.

Hou et al. [18] formulated a sheep to human Brucellosis transmission model that involved the human and sheep populations in Inner Mongolia, China. The model divided sheep population into susceptible, exposed and infectious while the human population was classified into susceptible, acute infection and chronic infection. The average bacterial load enough to cause infection on the host was defined as the infectious unit. Computation of the threshold number used in determination for the existence and stability of the equilibria was done. Numerical simulations and sensitivity analysis of the reproductive number were carried out using reported human Brucellosis data from the region. Findings showed that the disease could not be eliminated even if disinfection and immunization rates of adult sheep were 100\%. The study further investigated and compared the effects of immunization, disinfection and elimination strategies. Findings showed that vaccination and disinfection of both adult and young sheep were effective and appropriate control strategies for Brucellosis control in Inner Mongolia. However, an assumption that the exposed and infectious sheep had the same infectivity and shedding 
A Review of the Mathematical Models for Brucellosis Infectiology and Control Strategies

rates of Brucella to the environment through abortions or secretions makes the exposed class redundant. Similarly, the lack of data for human to human transmission was not a sufficient condition for this particular transmission route to be ignored.

Racloz et al. [19] performed a formal mathematical analysis of the Mongolia model by Zinsstag et al. [20] to examine different settings by which the number of latently infected, infectious animals remains stable. Although the need for more data was evident in order to have a better estimation of the threshold densities for the disease transmission in other regions of the world, the employed system of differential equations and the equilibrium conditions for the model were found to be reasonably robust. Demographic determinants for livestock were found to be the most important parameters for the disease persistence. The study further depicted that despite varying control efforts in pastoral areas, Brucellosis remains largely persistent worldwide. The study recommended for ecological considerations like sustaining ecosystem services when planning for the disease control strategies in pastoral settings. Improved governance, land reform, placing limitations on livestock stocking density, movement, and integrated social and economic development should be part and parcel of the plan. However, wild animals and the seasonal interactions between wild and domestic animals were not taken into account on the model formulation and analysis.

Li, Sun, Zhang, Jin, Sun, Wang, Huang and Zhang [21] presented a mathematical model to explore the effective control and preventive measures of Brucellosis transmission dynamics in Hinggan League of Inner Mongolia, China. The formulated model described the sheep-to-human and sheep-to-sheep spread of the disease. The sheep flock at any time was classified into basic ewes and other sheep, each of the classes was further divided into susceptible, recessive infected, quarantined seropositive infected and vaccinated subgroups while the human population was divided into susceptible, acute infections and chronic infections. The study indicated that susceptible sheep and human could acquire infections from polluted environment and recessive infected sheep. Numerical simulations for the study agreed with the 2001 to 2011 records of human Brucellosis incidences, and the trend of the disease incidences in was given. The reproduction number for the region was estimated to be 1.9789 . The study demonstrated that a combination of detection and elimination, vaccination, and prohibition of mixed feeding between basic ewes and other sheep are useful in controlling the disease in the region. However, the model does not fit to the sub-Saharan Africa countries where cattle, small ruminants, and wild animals share grazing areas and water points. An assumption that recessive infected and quarantined seropositive infected sheep release the same quantity of Brucella organisms into the environment per unit time makes the quarantined class redundant. Thus, there is a need for combining the recessive infected and quarantined seropositive infected classes.

Kang et al. [22] developed SIR model to simulate the spread of Brucellosis within the cattle population in India. The formulated model was used to estimate the impact of test-and-slaughter, reduction of the transmission rate, and mass vaccination in controlling the spread of the disease. The epidemiological benefits of different rates of vaccination and decreased transmission were analyzed. Findings showed that test-andslaughter is an effective strategy for eliminating and eradicating Brucellosis. However, socio-cultural restrictions prevents the culling of cattle in India. The model revealed 
Nkuba Nyerere, Livingstone S. Luboobi, Saul C. Mpeshe, and Gabriel M. Shirima

further that the reduction of Brucellosis transmission rates correspondingly lowered the stability and endemic levels of Brucellosis prevalence. Pulse vaccination initially lowered the prevalence rates but increased with the influx of new susceptible births. However, limitations in surveillance data acted as the major constraint of this study. In real practice, test and slaughter alone results in more financial losses than did eradication because the newborns are not protected. On the other hand, the model formulation was dictated by the available data and not the biology of the disease and so did not incorporate small ruminants, human population and the indirect route of disease transmission through the contaminated environment.

Nie et el. [23] described the spread of Brucellosis among dairy cattle in Jilin Province, China using a mathematical model. The dairy cattle population was classified into Susceptible, Exposed, and Infected while the bacterial compartment was included to capture the outside transferred amount of Brucella. The basic reproductive number was computed and used to prove the existence and global attraction of the equilibria. Parameter values for the system and prediction of infection numbers with time were estimated using 20 years Jilin province Brucellosis data. The findings from the study revealed that the disease would still persist in area for the next 30 years even if the existing measures were taken into account. Moreover, the combination of culling, sterilizing and minimization of the number of outer importing is the best control strategy for dairy cattle Brucellosis. However, the model did not consider the impact of small ruminants and weather variation on the disease spread.

Zhang et al. [4] presented a SEIV model to excavate the transmission of Brucellosis in Zhejiang Province in China. The formulated model fitted the real disease situation and predicted the disease tendency in the region. Assessment of the effectiveness of the control measures was done in dairy cows. Careful analysis of the model gave the following quantitative results: importation of dairy cows from northern areas limits control of the disease due to high fluctuation in the number of infectious dairy cows, and the rate of Brucella transmission from the environment to dairy cattle was greater than that from infectious dairy cattle to susceptible cattle. Brucellosis control strategies require consideration of seasonal parameters due to periodic nature under certain circumstances. Additionally, a combination of birth rate management and twice a week sterilization in infected regions best controls the disease in cows. Nevertheless, the model did not consider human and other animal populations.

$\mathrm{Li}$, Sun, Wu, Zhang and Jin [24] proposed SEIRV multi-group model incorporating two-way transmissions between cattle and sheep in public farms. The influence of bidirectional infection resulting from mixed feeding of the two groups was investigated. Investigation and confirmation for the uniform existence of a unique positive equilibrium were done using the computed basic reproduction number. An endemic equilibrium of the model was proved to be globally attracting if the basic reproduction number is $>1$. Sensitivity analysis of the infectious herds with different systems in terms of some parameter values was performed. Findings revealed that the disease cannot be eradicated if there is a two-way transmission between cattle and sheep; reproduction number been less than unity is not a sufficient condition for the disease eradication in the region. The study recommended that mixed feeding prohibition is the best control measure for Brucellosis elimination. However, the assumption that individuals at the latent stage and the one at the infectious stage have the same 

A Review of the Mathematical Models for Brucellosis Infectiology and Control
Strategies

transmission rate makes the exposed class redundant. In addition, the model did not incorporate the human population; a population that makes Brucellosis a public health concern.

Hou et al. [25] used Changling County of Jilin Province Brucellosis infection characteristics monitoring data to formulate a multi-stage model for the disease spread in sheep. Appertaining to the assumption that Brucella spread primarily causes disease in adult sheep and that young sheep infectivity is negligible, the sheep population was classified into young susceptible, susceptible adult or sexually mature, vaccinated, exposed and infectious classes. Basic reproductive number computation was done and the model dynamic properties were discussed. In terms of the embedded parameters, sensitivity analysis of the basic reproductive number was performed and found that the sheep birth, vaccination, and elimination rates for infectious sheep were important players in the disease transmission. Further investigation and comparison of the effects of sheep vaccination and culling strategies revealed that the two controls are effective and feasible for controlling Brucellosis in the region; however, the latter was more effective than the former. Nonetheless, the model did not consider the impact of the contaminated environment and human to human transmission in the dynamics of the disease.

Lou et al. [26] proposed a susceptible-exposed-infected-vaccinated (SEIV) Brucellosis model with periodicity in the rates of transmission. The impacts of seasonal disease transmission in livestock and human populations of Bayingolin Mongol Autonomous Prefecture of Xinjiang, China were investigated. Computation of the basic reproductive number was done and it was estimated to be 2.5524 . Sensitivity analysis of the reproductive number and the new acute human Brucellosis cases for the model parameters demonstrated that livestock reduced birth rate, increased slaughter rate of seropositive livestock, increased immunization rates for susceptible livestock, and decreased loss rates of immunization efficacy are effective strategies for controlling the Brucellosis epidemic. The study predicted a continuous increase in the number of newly acute human Brucellosis and its maximum value was estimated to be 15325 , which will be reached around the summer of 2023. However, this study did not consider the contribution of the contaminated environment in the disease transmission dynamics.

de Souza et al. [27] formulated a Brucellosis model for the purpose of measuring the impact of the combination of S19 and RB51 vaccines in reducing the disease prevalence. The model divided the cattle population into seven compartments namely: susceptible, vaccinated with the RB51 strain, primiparous latent carriers, vaccinated with the S19 strain, primiparous infectious cows, multiparous latent carriers, and multiparous infectious cows. The study concluded that the adoption of RB51 vaccination as a complement to S19 vaccination significantly reduces bovine Brucellosis prevalence in a short time. However, ignoring the contribution of small ruminants, wild animals, contaminated environment and the aspect of seasonality limits a better understanding of the disease transmission dynamics.

Li, Sun, Zhang and Jin [28] formulated a sheep and human Brucellosis dynamical model with direct and indirect transmission routes. The sheep population was grouped into basic ewes and other sheep and it was further divided into susceptible, recessive infected, quarantined seropositive infected and vaccinated subgroups while the human population was classified into susceptible, acute infections and chronic infections. 
Nkuba Nyerere, Livingstone S. Luboobi, Saul C. Mpeshe, and Gabriel M. Shirima

Recessive infected sheep and contaminated environment were the two main sources of infection for susceptible humans and sheep. It had been proved from this study that, Brucellosis extinction and persistence equilibria are globally attracting if and respectively. Nevertheless, this model did not consider goat and cattle populations which are mostly found in areas where sheep are kept.

$\mathrm{Li}$, Guo and Zhang [29] presented a mathematical model incorporating indirect transmission routes to evaluate animal vaccination, elimination of infected animals, and environmental disinfection control strategies. The study used national human data from eleven China provinces that have a high number of Brucellosis cases to compare results for three different models. Potential possible disease outbreaks were investigated using the model with the best fit and standard incidence. It was found that the country's average reproduction number was relatively less than that of the province with high disease incidence, suggesting that indirect transmission of the disease was a more common route compared to direct transmission. The study concluded that Brucellosis in China could be controlled if infected animals elimination, environmental disinfection, and animal vaccination ware increased. The findings further suggested that the combination of infected animals' elimination, environmental disinfection, and animal vaccination is necessary for ensuring cost-effective Brucellosis control scheme. However, the important aspects of the disease control like personal protection in humans, and the impacts of seasonal weather variations were not captured in the model.

Tumwiine and Robert [30] presented a mathematical model depicting the conveyance of Brucellosis in the bovine herd. The model analysis was carried out to gain and establish the stability of the equilibria. A boundary parameter categorized as the basic reproduction number was calculated and the conditions under which bovine Brucellosis can be cleared in the cattle population were established. It was found out that if $R_{0}<1$, the infection can be eliminated in the cattle population or persists if $R_{0}>1$. Lyapunov function and Poincair'e-Bendixson theory were used to prove that the disease extinction and persistence equilibria are respectively globally asymptotic stable. Numerical simulation revealed that an increase in magnitude of treatment rate for infected cattle and reduction of contact rate between infectious cattle and susceptible or recovered cattle controls the disease. However, the formulated model can be improved by incorporating small ruminants and the aspect of seasonality that captures cattle calving season, temperature, humidity, soil salinity, and exposure of the bacteria to sunlight.

Zhou et al. [31] developed a multi-group model to explore the key factors, potential effects, characterize Brucellosis transmission, and prioritize control measures. Direct Lyapunov method and asymptotic autonomous systems theory were used to characterize the global threshold dynamics of the disease. The weighted sum method was used in the formulation of a multi-objective optimization problem, and it was converted to a scalar optimization problem of minimizing the total cost for control. Pontryagin's Maximum Principle was used to establish the existence and characterization of an optimal control problem. Model parameterization and computation of optimal control strategy for Inner Mongolia in China were done. The effects of health education on human, sheep recruitment, culling of infected sheep, and sheep vaccination to the dynamics and control of the disease were explored. The study revealed that Brucellosis would continue to increase in Inner Mongolia because the available controls were not working well. The controls recommended in this study are health education, sheep 
A Review of the Mathematical Models for Brucellosis Infectiology and Control Strategies

vaccination and restriction of unregulated sheep breeding. However, the model did not consider the contribution of other ruminants and the impact of seasonal weather variations on the disease transmission.

Nepomuceno et al. [32] presented a network type of individual-based modeling as an alternative approach to compartmental models that are used in controlling bovine Brucellosis. Spatial aspects like migration between herds, heterogeneous populations, and control actions designated as pulse interventions were considered and implemented in the model. It was demonstrated that the mean-field behaviour of a compartmental model could be reproduced by an equivalent individual-based model. To enable the replication of the presented results, details of this process and flowcharts were provided. Real parameters from Sao Paulo state of Brazil herds were used to investigate three numerical examples in situations that explore meta-population, pulsed and continuous vaccination, and eradication effects. The analysis from this study depicted results which agreed with the expected disease behaviour.

Abatih et al. [33] presented a mathematical approach to analyze the transmission dynamics of Brucellosis among bison. Quantitative and qualitative analyses were used to show that the infection would disappear from the herd if $R_{0}<1$ and will persist otherwise. Results from Sobol method for global sensitivity analysis showed that recovery rate and loss of resistance rate were accountable for the high inconsistency in the expected number of infectious bison. Partial ranked correlation coefficients computation revealed that transmission coefficient, recovery rate, mortality rate, and density-dependent reduction in birth correlates highly negatively with the infective bison number while the calving rate and resistance loss rate highly correlates positively with the infectious bison number. Thus, measures to control the disease in bison should target at rising the size of mortality rate, recovery rate, and birth reduction density-dependent rate as well as decreasing calving rate and loss of resistance. To raise the accuracy of the expected number infectious bison, a precise estimate of the recovery rate and loss of resistance from experimental studies are needed. However, the recommendations from this study are socially and economically inapplicable.

Lolika et al. [34] introduced a framework for Brucellosis mathematical modeling that aimed at improving the quantitative understanding of the disease dynamics. In particular, the framework introduced which is an extension of the model by [33] was used to explore the impact of culling control and the influence of chronic individuals on the Brucellosis spread in the periodic or non-periodic environment. The model was analyzed to get insights on the epidemic and endemic behaviour of the disease using threshold dynamics described by the basic reproduction number. Culling at an optimal level in a periodic and non-periodic environment was explored using optimal control theory. However, this study did not incorporate the indirect transmission of the disease.

\section{Summary}

Mathematical models presented by [17, 25] investigated the transmission dynamics of Brucellosis in a population of sheep only while those presented by [4, 16, 22, 23, 27] focused on the dynamics of the disease in a population of cattle only. These models missed important aspects of the disease behaviour; Brucellosis is a zoonosis; the population of humans cannot be easily ignored when studying the disease dynamics. On 
Nkuba Nyerere, Livingstone S. Luboobi, Saul C. Mpeshe, and Gabriel M. Shirima

the other hand, the study by [34] adopting the study by [33] explored the impacts of various parameters accountable for transmission of the disease in a population of bison. However, the study is more theoretical as it considers a single population and does not show the effect of bison Brucellosis to other populations or the environment.

Furthermore, mathematical models by $[18,21,28]$ were formulated to investigate the impact of various control strategies on sheep and human populations. The inclusion of humans, sheep and the contaminated environment in model and the incorporated control strategies provides a better understanding of Brucellosis dynamics compared to the ones with one or two populations. Nevertheless, the models could be improved by incorporating other ruminant populations and seasonality in the transmission of the disease. A mathematical model by [23] takes consideration of cattle and sheep populations and investigates the impact of mixed feeding and bidirectional infection between the two populations. This mathematical model is far better and can be improved more by including the human population and other domestic ruminants like goats so as to fit in most of the pastoral communities. Seasonal weather variation parameters and wild animals can also be incorporated so as to have a more realistic model. The [20] mathematical formulation adopted by [19] is also a better model as it includes sheep, goat and human populations, and it provides a better understanding of the disease dynamics as it incorporates most of the transmission key players. However, the model can be improved by incorporating the indirect route of transmission, seasonal weather parameters for disease dynamics and wild animals.

In terms of control strategies six of the mathematical models $[4,16,17,23,27$, $32]$ investigated the use of one control measure, five $[4,18,22,25,26]$ explored the impact of the combination of two controls while four studies [21, 23, 29, 31] examined the impact of a combination of three control strategies in minimizing or elimination of the disease. The remaining studies aimed at providing potential information for Brucellosis spread. Based on the model structure and control strategies incorporated, the model by [29] fits in areas with mixed farming systems. However, it can be improved by considering personal protection in humans, wild animals, and seasonality. In addition, the mathematical model proposed by [18] and [25] are recommended for studying the disease in a single animal population and can also be improved by incorporating controls like personal protection, and environmental hygiene and sanitation.

\section{Conclusions}

Diversities have been observed in the reviewed mathematical models; some models incorporate seasonal variations in a single animal population, some ignored the contributions of the contaminated environment while others considered only the cattle or sheep population. Most of the models did not consider the contribution of wild animals and personal protection control in the dynamics of Brucellosis whereas few of them incorporated cattle and sheep only. In addition, some studies did not match the real situation for most Brucellosis endemic areas like sub-Saharan African region and Asian countries where wild animals, cattle and small ruminants share grazing areas and water points. In Brucellosis endemic areas, models with at least three populations and the combination of at least two control parameters works better. Generally, this review revealed that mathematical models regardless of their goodness cannot be applied everywhere; not all mathematical models fit every setting or group of animals. Some 
A Review of the Mathematical Models for Brucellosis Infectiology and Control Strategies

models provide more insights on the disease dynamics and control in developed countries while others give a better understanding of the disease and control in developing countries. This review recommends that mathematical models by [20] and [29] though not perfect may be used in sub Saharan Africa and other areas where mixed farming is practiced.

Acknowledgements. Authors acknowledgments the financial support from African Development Bank (AfDB). Also, we are thankful to the referees for their valuable report for the improvement of the paper.

\section{REFERENCES}

1. H.V.Wyatt, Lessons from the history of brucellosis, Journal of Maltese History, 5(1) (2016) 75-84.

2. O.Akpinar, Historical perspective of brucellosis: a microbiological and epidemiological overview, Le Infezioni in Medicina, 24(1) (2016) 77-86.

3. M.J.Mutolo, L.L.Jenny, A.R.Buszek, T.W.Fenton and D.R.Foran, Osteological and molecular identification of brucellosis in ancient butrint, Albania, American Journal of Physical Anthropology, 147(2) (2016) 254-263.

4. J. Zhang, G.-Q.Sun, X.-D.Sun, Q.Hou, M.Li, B.Huang, H.Wang and Z.Jin. Prediction and control of brucellosis transmission of dairy cattle in zhejiang province, China. Plos one. 9(11): 1-13. 2014.

5. A.El-Sayed and W.Awad, Brucellosis: Evolution and expected comeback, International Journal of Veterinary Science and Medicine, 6(1) (2018) 31-35.

6. J. Godfroid, A.Cloeckaert, J.P.Liautard, S.Kohler, D.Fretin, K.Walravens, B.GarinBastuji and J.J.Letesson, From the discovery of the malta fever's agent to the discovery of a marine mammal reservoir, brucellosis has continuously been a reemerging zoonosis, Veterinary Research, 36(3) (2005) 313-326.

7. WHO. Brucellosis. Fact sheet N173. World Health Organization, Geneva, Switzerland, 1997.

8. G.C.Bishop, P.P.Bosman and S.Herr, Bovine brucellosis, Infectious Diseases of Livestock with Special Reference to Southern Africa, 2 (1994) 1053-1066.

9. WHO. The Development of new/improved brucellosis vaccines: report of a WHO meeting, Geneva, Switzerland, 11-12 December 1997 (No. WHO/EMC/ZDI/98.14). Geneva: World Health Organization. 1998.

10. G.Pappas, P.Papadimitriou, N.Akritidis, L.Christou and E.V.Tsianos, The new global map of human brucellosis, The Lancet Infectious Diseases, 6(2) (2006) 91-99.

11. D.Bernoulli and S.Blower, An attempt at a new analysis of the mortality caused by smallpox and of the advantages of inoculation to prevent it, Reviews in Medical Virology, 14(5) (2004) 275-288.

12. C.I.Siettos and L.Russo, Mathematical modeling of infectious disease dynamics, Virulence, 4(4) (2013) 295-306.

13. F.Brauer and C.Castillo-Chavez, Mathematical Models for Communicable Diseases., Vol. 84. SIAM. 2013.

14. D.L.Smith, K.E.Battle, S.I.Hay, C.M.Barker, T.W.F.Scott and E.McKenzie, Ross, macdonald, and a theory for the dynamics and control of mosquito-transmitted 
Nkuba Nyerere, Livingstone S. Luboobi, Saul C. Mpeshe, and Gabriel M. Shirima pathogens. PLoS pathogens. 8(4): e1002588. doi:10.1371/journal.ppat.1002588. 2004.

15. H.W.Hethcote, A thousand and one epidemic models. In: Frontiers in mathematical biology. Springer. 1994, 504-515.

16. M.Amaku, R. Dias, J.Ferreira Neto and F.Ferreira, Mathematical modeling of bovine brucellosis control by vaccination, Arquivo Brasileiro de Medicina Veterin'ariae Zootecnia, 61 (2009) 135-141.

17. B.Ainseba, C.Benosman, and P.Magal, A model for ovine brucellosis incorporating direct and indirect transmission, Journal of Biological Dynamics, 4(1) (2010) 2-11.

18. Q.Hou, X.Sun, J.Zhang, Y.Liu, Y.Wang and Z.Jin, Modeling the transmission dynamics of sheep brucellosis in Inner Mongolia autonomous region, China, Mathematical Biosciences, 242(1) (2013) 51-58.

19. V.Racloz, E.Schelling, N.Chitnis, F.Roth and J.Zinsstag, Persistence of brucellosis in pastoral systems, OIE Revue Scientifique et Technique, 32(1) (2013) 61-70.

20. J.Zinsstag, F.Roth, D.Orkhon, G.Chimed-Ochir, M.Nansalmaa, J.Kolar and P.Vounatsou, A model of animal-human brucellosis transmission in Mongolia, Preventive Veterinary Medicine, 69 (1-2) (2005) 77-95.

21. M.Li, G.Sun, J.Zhang, Z.Jin, X.Sun, Y.M.Wang, B.Huang and Y.Zheng, Transmission dynamics and control for a brucellosis model in Hinggan League of Inner Mongolia, China, Mathematical Bioscience and Engineering, 11(5) (2014) 1115-1137.

22. G.J.Kang, L.Gunaseelan and K.M.Abbas, Epidemiological modeling of bovine brucellosis in India. In: 2014 IEEE International Conference on Big Data (Big Data). IEEE. pp. 6-10, 2014.

23. J.Nie, G.-Q.Sun, X.-D.Sun, J.Zhang, N.Wang, Y.-M.Wang, C.-J.Shen, B.-X.Huang and Z.Jin, Modeling the transmission dynamics of dairy cattle brucellosis in jilin province, China, Journal of Biological Systems, 22(4) (2014) 533-554.

24. M.-T.Li, G.-Q.Sun, Y.-F.Wu, J. Zhang and Z.Jin, Transmission dynamics of a multigroup brucellosis model with mixed cross infection in public farm, Applied Mathematics and Computation, 237 (2014) 582-594.

25. Q.Hou and X.-D.Sun, Modeling sheep brucellosis transmission with a multi-stage model in changling county of jilin province, China, Journal of Applied Mathematics and Computing, 51(1-2) (2016) 227-244.

26. P.Lou, L.X.Zhang, J.Xu and K.Wang, Modelling seasonal brucellosis epidemics in bayingolin mongol autonomous prefecture of Xinjiang, China, 2010-2014, BioMed Research International, (2016) 1-17.

27. V.A.F.de Souza, J.S.F.Neto, M.Amaku, R.A.Dias, E.O.Telles, J.H.H.Grisi-Filho, M.B.Heinemann and F.Ferreira, Mathematical modeling of bovine brucellosis control using the rb51 vaccine, Semina: Ciências Agrárias, 37(5) (2016) 3767-3775.

28. M.-T.Li, G.-Q.Sun, W.-Y.Zhang and Z.Jin, Model-based evaluation of strategies to control brucellosis in China, International Journal of Environmental Research and Public Health, 14(3) (2017) 1-15.

29. C.Li, Z.-G.Guo and Z.-Y.Zhang, Transmission dynamics of a brucellosis model: Basic reproduction number and global analysis, Chaos, Solitons \& Fractals, 104 (2017) 161-172. 
A Review of the Mathematical Models for Brucellosis Infectiology and Control Strategies

30. J.Tumwiine and G.Robert, A mathematical model for treatment of bovine brucellosis in cattle population, Journal of Mathematical Modeling, 5(2) (2017) 137-152.

31. L.Zhou, M.Fan, Q.Hou, Z.Jin and X.Sun. Transmission dynamics and optimal control of brucellosis in Inner Mongolia of China, Mathematical Biosciences and Engineering, 15(2) (2018) 543-567.

32. E.G.Nepomuceno, A.M.Barbosa, M.X.Silva and M. Perc, Individual-based modelling and control of bovine brucellosis, Royal Society Open Sscience, 5(5) (2018) 180-200.

33. E.Abatih, L.Ron, N.Speybroeck, B.Williams and D.Berkvens, Mathematical analysis of the transmission dynamics of brucellosis among bison, Mathematical Methods in the Applied Sciences, 38(17) (2015) 3818-3832.

34. P.O.Lolika, C.Modnak and S.Mushayabasa, On the dynamics of brucellosis infection in bison population with vertical transmission and culling, Mathematical Biosciences, 305 (2018) 42-54. 